ARTICLE

Received 16 Dec 2014 | Accepted 26 Mar 2015 | Published 28 Apr 2015

DOI: $10.1038 /$ ncomms 8048

\title{
Transcriptional activation by the thyroid hormone receptor through ligand-dependent receptor recruitment and chromatin remodelling
}

\author{
Lars Grøntved ${ }^{1,2}$, Joshua J. Waterfall ${ }^{3}$, Dong Wook Kim4, Songjoon Baek, Myong-Hee Sung ${ }^{1}$, Li Zhao ${ }^{4}$, \\ Jeong Won Park ${ }^{4}$, Ronni Nielsen², Robert L. Walker ${ }^{3}$, Yuelin J. Zhu ${ }^{3}$, Paul S. Meltzer ${ }^{3}$, Gordon L. Hager ${ }^{1}$ \\ \& Sheue-yann Cheng ${ }^{4}$
}

\begin{abstract}
A bimodal switch model is widely used to describe transcriptional regulation by the thyroid hormone receptor (TR). In this model, the unliganded TR forms stable, chromatin-bound complexes with transcriptional co-repressors to repress transcription. Binding of hormone dissociates co-repressors and facilitates recruitment of co-activators to activate transcription. Here we show that in addition to hormone-independent TR occupancy, ChIP-seq against endogenous TR in mouse liver tissue demonstrates considerable hormone-induced TR recruitment to chromatin associated with chromatin remodelling and activated gene transcription. Genome-wide footprinting analysis using DNase-seq provides little evidence for TR footprints both in the absence and presence of hormone, suggesting that unliganded TR engagement with repressive complexes on chromatin is, similar to activating receptor complexes, a highly dynamic process. This dynamic and ligand-dependent interaction with chromatin is likely shared by all steroid hormone receptors regardless of their capacity to repress transcription in the absence of ligand.
\end{abstract}

\footnotetext{
${ }^{1}$ Laboratory of Receptor Biology and Gene Expression, Center for Cancer Research (CCR), National Cancer Institute (NCI), National Institutes of Health (NIH), Bethesda, Maryland 20892, USA. ${ }^{2}$ Department of Biochemistry and Molecular Biology, University of Southern Denmark, 5230 Odense, Denmark. ${ }^{3}$ Genetics Branch, CCR, NCI, NIH, Bethesda, Maryland 20892, USA. ${ }^{4}$ Laboratory of Molecular Biology, CCR, NCI, NIH, Building 37/NIH, Bethesda, Maryland 20892, USA. Correspondence and requests for materials should be addressed to S.C. (email: chengs@mail.nih.gov).
} 
S ynthetic thyroid hormones are one of the most prescribed drugs worldwide to treat hypothyroidism. The thyroid hormone receptor (TR) belongs to the steroid hormone receptor family and exists as two different subtypes (TR $\alpha$ and TR $\beta$ ) encoded by different genes and each in multiple thyroid hormone (T3)-binding and non-T3-binding isoforms ${ }^{1}$. Each subtype is expressed in a tissue-specific manner. TR $\beta 1$ is predominantly expressed in kidneys, liver, brain, heart and thyroid, while TR $\alpha$ is expressed at highest level in brain ${ }^{1}$. For simplicity, we will refer to T3-binding TR isoforms in the liver as TR. TR shares many structural and functional similarities with other members of the receptor family. However, in contrast to receptors such progesterone receptor (PR) and glucocorticoid receptor (GR), unliganded TR localizes primarily to the nucleus ${ }^{2}$ and interacts with nucleosome-embedded $\mathrm{DNA}^{3}$. Unliganded, chromatin-bound TR represses transcription through direct interaction with transcriptional co-repressors such as SMRT and $\mathrm{NCoR}^{4-6}$. Binding of hormone is thought to induce a conformational change of TR, causing displacement of co-repressors and recruitment of co-activators ${ }^{7-10}$. This relatively simple bimodal switch, initially described in the late nineties, results in a transition from gene repression to gene activation ${ }^{11}$. Currently, this model is generally accepted for a basic understanding of TR-regulated gene transcription (for recent reviews see, for example, refs 12-14) and also discussed as a general principle for nuclear receptors and other transcription factors $(\mathrm{TFs})^{15,16}$.

We have previously shown that genomic scale studies using a combination of DNase-seq and chromatin immunoprecipitation sequencing (ChIP-Seq) inform our understanding of steroid hormone receptors interaction with chromatin ${ }^{17-20}$; however, such data is currently unavailable for endogenous TR. Here we use ChIP-seq to identify endogenous TR-binding sites (TRBS) genome wide in mouse liver tissue and DNase-seq to map chromatin remodelling promoted by thyroid hormone. We confirm that the unliganded TR indeed interacts with a number of regions in the genome. However, in contrast to the bimodal switch model, we find evidence for considerable ligand-dependent TR recruitment to the genome and de novo remodelling of chromatin. Importantly, many thyroid hormone-regulated genes are associated with this mechanism of action. Moreover, in disagreement with models depicting $\mathrm{TR}$ as a repressor constitutively bound to chromatin in the absence of hormone, we find little evidence for TR footprints (FPs) genome wide, either in the presence or absence of ligand, suggesting that TR interaction with chromatin is generally highly dynamic. Thus, in addition to the ligand-dependent switch of transcriptional coregulators recruited to chromatin-bound TR, we propose a model wherein TR is able to dynamically bind and increase accessibility of chromatin in a ligand-dependent manner.

\section{Results}

Thyroid hormone regulation of chromatin accessibility. To understand the molecular consequences of genome-wide interaction of TR with chromatin, we mapped the DNase I accessible chromatin landscape in liver from mice treated with propylthiouracil (PTU) mimicking hypothyroid conditions and PTU followed by L-triiodothyronine (T3) resembling hyperthyroidism. Nuclei were purified from freshly isolated livers and treated with DNase I as previously described ${ }^{21}$. Small mildly digested DNA fragments liberated from accessible chromatin were purified by sucrose gradients and initially sequenced to a depth of 20-30 million tags (Supplementary Table 1). Replicate concordant DNase hypersensitive sites (DHS) were identified as previously described ${ }^{17}$; between 65,000 and 73,000 DHSs were mapped in liver from hypo and hyperthyroid animals. Interestingly, we find thousands of regions where T3 treatment results in robust changes of chromatin accessibility (Fig. 1a). Sixty-nine per cent of the changes are modest but significant (remodelled DHS, Fig. 1a,b) and likely reflect a net gain or loss of TFs or a transition of co-regulator interaction with TFs at the individual remodelled regions. Importantly, in contrast to the current model of constitutive TR interaction with chromatin, we observe that $31 \%$ of the differentially regulated DHSs are either formed or erased, where half of these are generated de novo as a consequence of T3 treatment (de novo, Fig. 1a,b). Figure 1c illustrates representative categories of accessible regions of chromatin identified. The hormone-remodelled regions are primarily located within intronic and intergenic regions (Fig. 1d) in agreement with genome-wide hormone-dependent chromatin remodelling by other steroid hormone receptors ${ }^{18}$. De novo motif analysis of DHSs induced by T3 shows an enrichment of a DR4-like motif (Fig. 1e) closely resembling the canonical DR4-based TR response element (TRE) and matches the TRE reported recently based on ChIP-seq against tagged overexpressed $\mathrm{TR}^{22-24}$. In addition to the TRE, we also find enrichment of motifs for HNF6 and FOXA. We do not find any additional significant enrichment of palindromic or inverted repeats of the AGGTCA half site. Approximately half of the remodelled DHSs contain the DR4-based TRE (Fig. 1f), suggesting that TR directly interacts with DNA at these sites. Thus at de novo-remodelled TREs, TR may be ligand dependently recruited to chromatin. In contrast, the DHSs with T3-dependent reduced accessibility are not enriched for DR4 TRE motifs compared with unchanged accessible regions (Fig. 1f,g), implying either that TR is not interacting directly with DNA at these sites and they are remodelled by secondary factors or that TR is recruited by a mechanism independent of the DR4 motif. At the T3-reduced DHS, we found enrichment of motifs likely to bind a number of liver-enriched transcriptions factors such as HNF4, C/EBP and HNF1 (Supplementary Fig. 1a).

T3 regulates transcription and local chromatin remodelling. The findings shown in Fig. 1 clearly reveal that we have identified a number of regulatory regions in the genome that are dramatically remodelled by $\mathrm{T} 3$ treatment. To investigate whether these remodelled regions are relevant for T3-regulated gene transcription, we identified T3-regulated genes in liver under hypo or hyperthyroid conditions. Genome-wide regulation of T3-dependent transcription was analysed by Illumina expression arrays. We identified several known direct targets of TR such as Thrsp/Spot14 and Dio1 (arrows, Fig. 2a and Supplementary Table 2). We found no apparent changes of chromatin accessibility of regulatory regions near Thrsp (Fig. 2b, upper panel), a well-described target for TR in liver, consistent with co-factor switching as the mechanism of TR activity at this locus. Interestingly, however; transcriptional induction of Dio1 is associated with de novo and induced remodelling of several regulatory sites near the Dio1 promoter (Fig. 2b, middle panel). In fact, a significant number of T3-induced genes were associated with nearby T3-remodelled chromatin compared with T3-repressed genes (Fig. 2c) and more than half of T3-induced genes but less than a tenth of T3-repressed genes have at least one induced remodelled DHS region within $50 \mathrm{~kb}$ of the transcription start site (TSS) (Supplementary Fig. 1b). Also regions with increased accessibility after $\mathrm{T} 3$ treatment were significantly closer to TSSs of T3-induced genes compared with repressed DHSs (Fig. 2d,e). In contrast, T3-repressed genes, such as Agxt2ll, are more frequently associated with DHSs reduced in accessibility after T3 treatment (Fig. 2b,c and e and Supplementary Fig. 1b-d). Thus, TR regulation of gene expression was to a large extent 
associated with nearby remodelling, including de novo remodelling, of regulatory regions, suggesting that the transition from repressed to activated state might not simply work through a switch from co-repressor to co-activator interaction with pre-occupied TR. In addition, co-activators and chromatin remodellers may also be recruited through ligand-dependent TR occupancy of chromatin, a mechanism shared with other steroid hormone receptors such as GR, PR and AR.

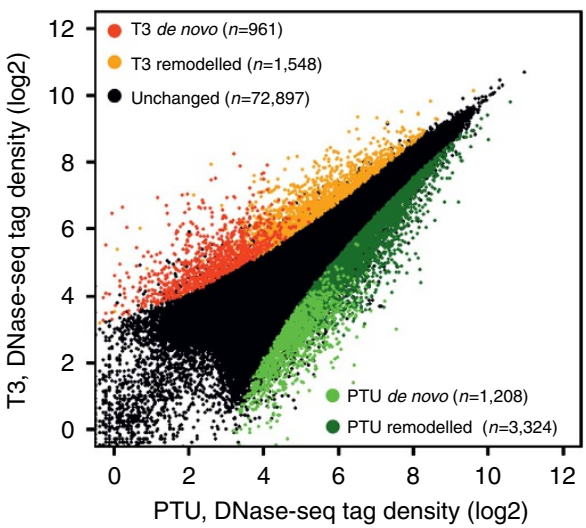

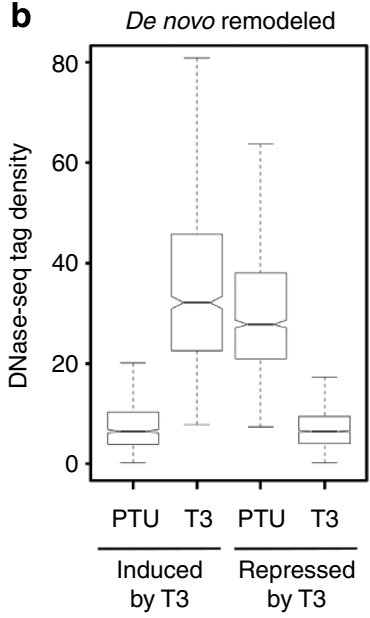

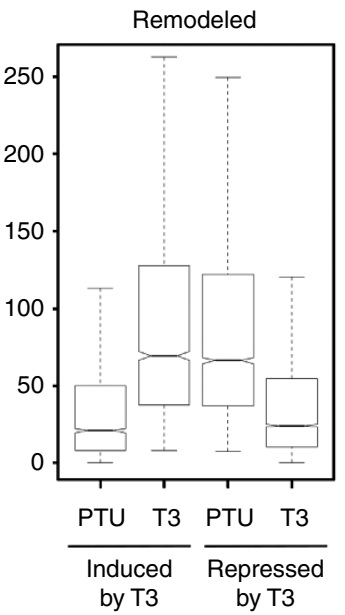

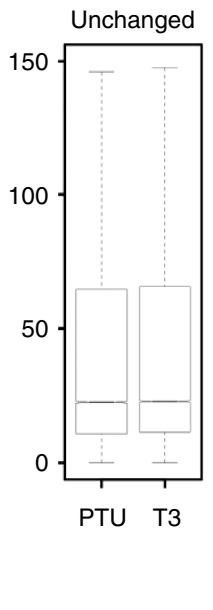
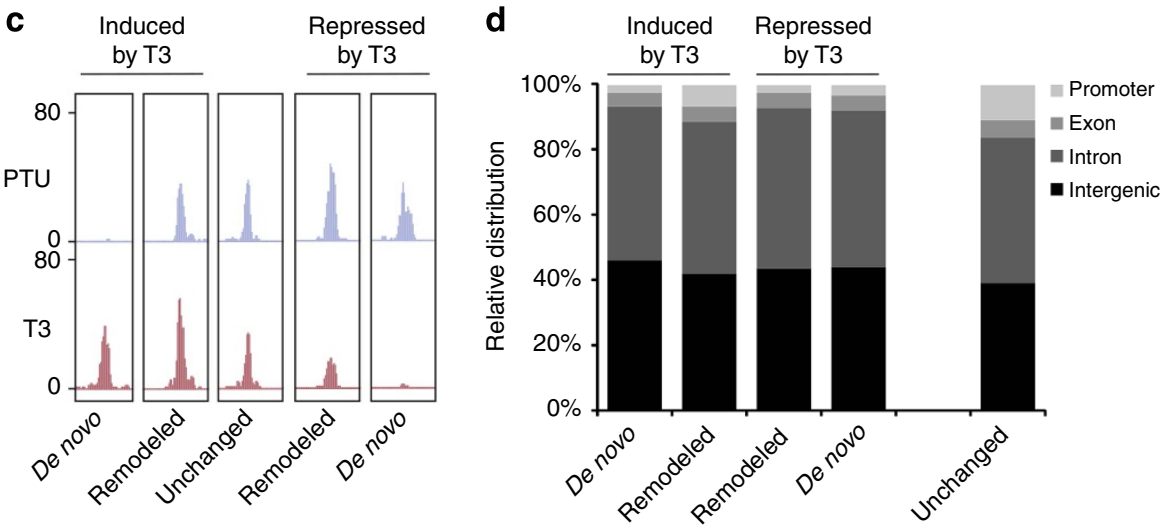

e

[००]

All T3 induced DHS

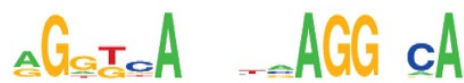

DR-4, TR response element (TRE), $P=1 \mathrm{e}-160$

T3 induced DHS without DR-4, TRE

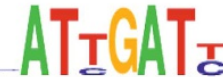

HNF6 motif, $P=1 \mathrm{e}-77$

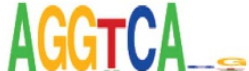

NR half site, $P=1 \mathrm{e}-52$

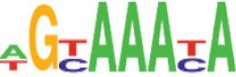

FOXA motif, $P=1 \mathrm{e}-28$

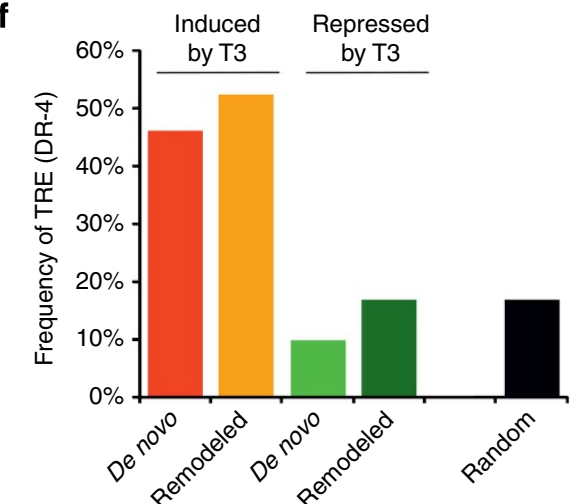

g

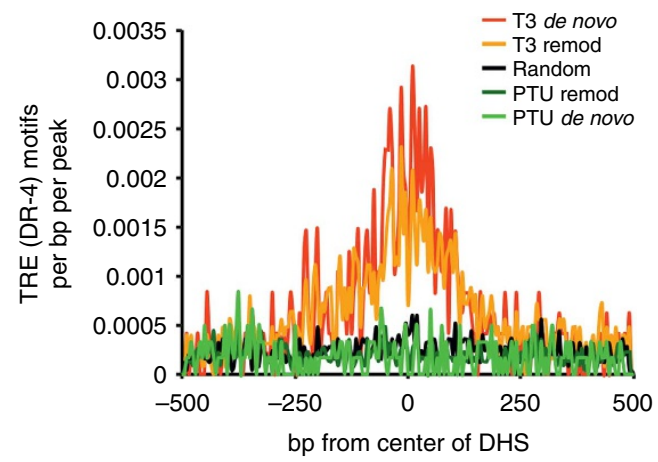

Figure 1 | Thyroid hormone induces de novo remodelling of chromatin in the liver. (a) DNase-seq tag density of accessible chromatin in liver of mice treated with PTU or PTU together with T3. Replicate concordant DHS hotpots were identified based on two biological replicates (average tag densities of DHSs from the two biological replicates are plotted). Red and orange data points represent DHS hotspots increased in accessibility as a consequence of T3 treatment, where red marks de novo DHSs. Dark and light green represent DHS hotspots with decreased accessibility after T3 treatment, where light green marks DHS that disappears as a consequence of hormone treatment. Black shows unchanged accessible regions. Differential DHS accessibility where identified at an adjusted $P$ value $<0.05$. (b) Distribution of DNase-seq tag density at de novo, remodelled and unchanged accessible regions. (c) Representative genome browser shots of DHS categorized as de novo, remodelled and unchanged. (d) Relative distribution of DHS within promoters ( $-1 \mathrm{~kb}$ to $+100 \mathrm{bp}$ of TSS), exons, introns and intergenic regions. (e) Upper panel, de novo motif analysis of all DHS with T3-induced DNase accessibility. The most enriched motif is shown. Lower panel, de novo motif analysis of T3-induced DHS lacking the identified canonical DR4-based thyroid hormone response elements (TRE). The three most enriched motifs are shown. (f) Frequency of the DR4 TRE at T3-induced and repressed accessible regions of chromatin. (g) Relative distribution of DR4 TRE in DHS hotspots, where accessibility is T3 regulated. 


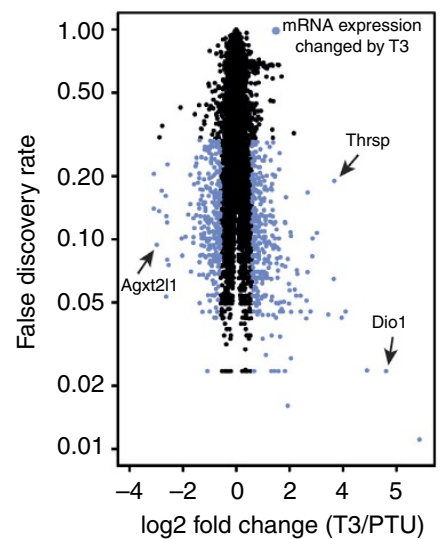

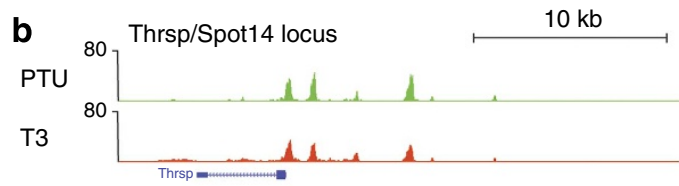
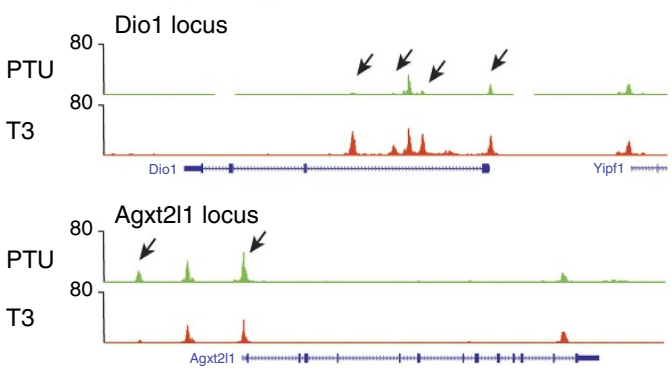
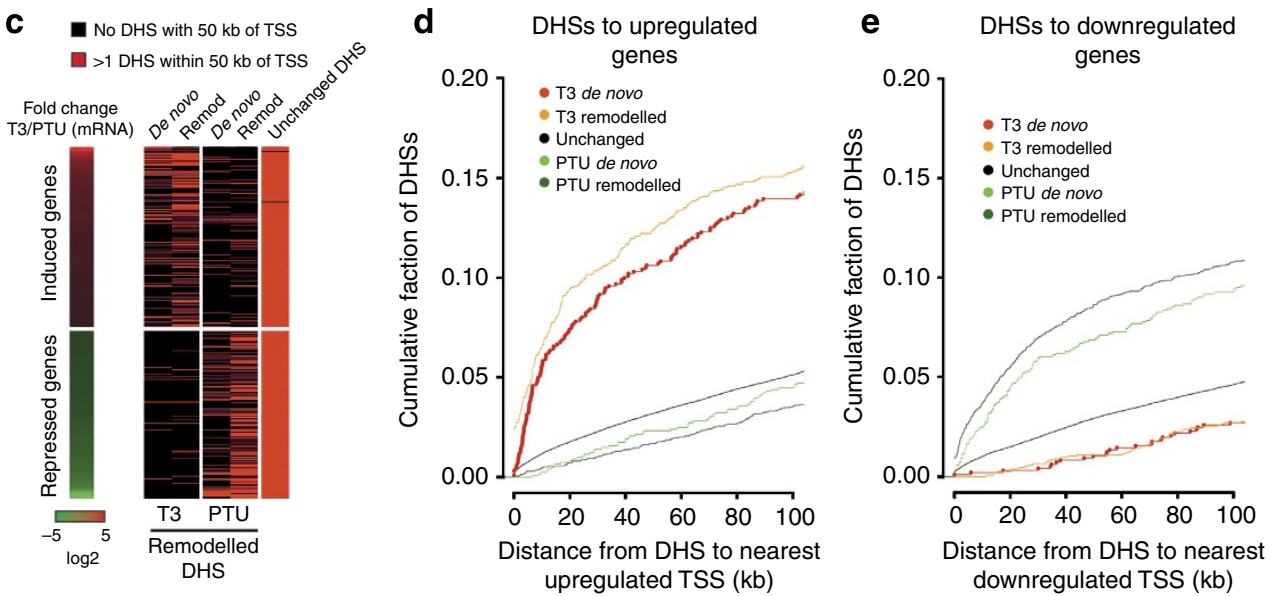

Figure 2 | Chromatin remodelling correlates with level of nearby gene transcription. (a) T3-induced and -repressed genes in the liver identified by mRNA expression microarrays of three independent biological repeats. Blue data points represent differentially expressed genes scored by an FDR of 0.3 and a log2 fold change of 1.5 (genes induced by T3) or -1.5 (genes repressed by T3). Two T3-induced genes (Thrsp and Dio1) and one T3-repressed gene (Agxt2/1) are marked by arrows. (b) Level of DNase accessibility near regions of T3-induced genes (Thrsp and Dio1) and a repressed gene (Agxt2/1). Arrows mark DHS accessibility regulated by T3. (c) Heatmap illustrating quantity of T3-regulated genes with at least one T3-regulated DHS within $50 \mathrm{~kb}$ of TSS. Genes are sorted from most induced to most repressed by T3. (d) Cumulative fraction of T3-regulated DHS near TSS (0-100 kb) of T3-induced genes. (e) Cumulative fraction of T3-regulated DHS near TSS (0-100 kb) of T3-repressed genes.

Identification of TRBS in liver tissue. To evaluate actual TR recruitment to chromatin genome wide at regions remodelled by T3, we applied ChIP-seq against endogenous TR. We used an in-house produced monoclonal antibody against the ligandbinding domain of TR $\beta$ (TR antibody C1). This antibody recognizes both $\operatorname{TR} \beta$ and $\operatorname{TR} \alpha$ (Supplementary Fig. 2). However, only $\operatorname{TR} \beta$, but not $\operatorname{TR} \alpha 1$ was detected in liver nuclear extracts by co-IP experiments (Supplementary Fig. 2a). To reduce the number of false positives in our ChIP-seq for TRBS, we performed a parallel ChIP-seq against TR in livers from mice with deficiency in both TR isoforms (TR double knockout (KO) mice). Enriched regions for TR occupancy were subsequently identified using TR double $\mathrm{KO}$ as background control and a stringent cutoff criterion for peak identification. We identified 864 TRBS under hypothyroid conditions and 2,186 under hyperthyroid conditions (Fig. 3a). Most ligand-independent TR peaks overlapped with TR peaks identified in presence of T3, generating a group of 651 preexisting TRBS. More than twice as many ligand-facilitated TRBS were found. De novo motif analysis of TRBS shows enrichment of the TRE closely resembling the TRE identified within T3-remodelled DHS (compare top panels of Figs $3 \mathrm{~b}$ and $1 \mathrm{e})$. Close to $60 \%$ of the preexisting and $42 \%$ of the hormonefacilitated TRBSs contain TREs (bottom panel, Fig. 3b), whereas $<20 \%$ of the TRBSs unique to hypothyroid conditions contain a
TRE, which is close to background levels of TREs in accessible chromatin (broken line in the bottom panel of Fig. 3b). The tag density of the TRBSs unique to hypothyroid conditions is also significantly lower than preexisting TRBSs (Supplementary Fig. 3a), suggesting that these peaks may either be indirect TRBSs (that is, tethered to other TFs) or false positives. In general, $92 \%$ of TRBSs localize to accessible chromatin (Supplementary Fig. 3b), in agreement with previous reports on steroid hormone receptor occupancy of the accessible genome $^{17,18,25}$.

Although we used stringent criteria for TR peak identification the number of identified TRBS is relative low compared with other nuclear receptors in mouse liver tissue (for example GR $>10,000$ binding sites ${ }^{17}$ ). Moreover, the detected TR only occupy $27 \%$ of the total T3 remodelled and $14 \%$ of the de novoremodelled DHS (Supplementary Fig. 3c), collectively implying that the monoclonal TR antibody used here could fail to immunoprecipitate the total pool of TR bound to chromatin. To confirm TR occupancy of chromatin, we probed genome-wide binding of $\mathrm{RXR} \alpha$, reported to be the most frequent heterodimerization partner of TR in liver ${ }^{26}$. A total of $41 \%$ of T3-remodelled DHS and $24 \%$ of T3 de novo-remodelled chromatin is occupied by RXR (Supplementary Fig. 3c), suggesting that the monoclonal TR antibody may miss a 
a

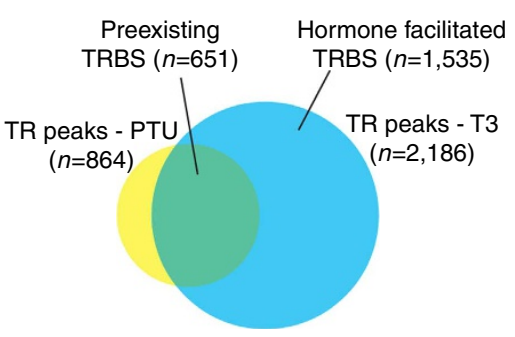

b

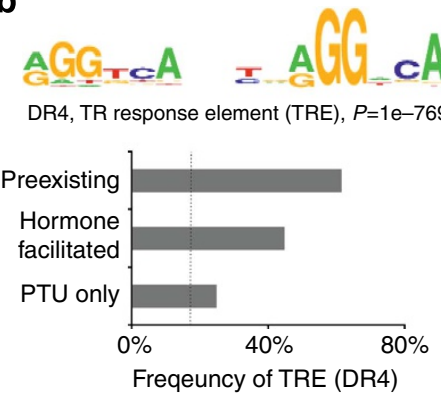

C

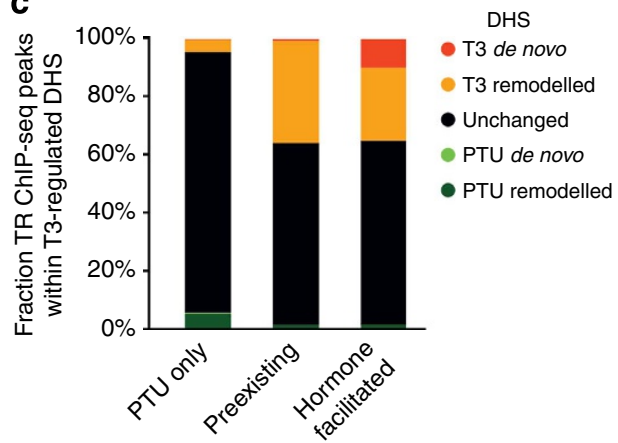

d
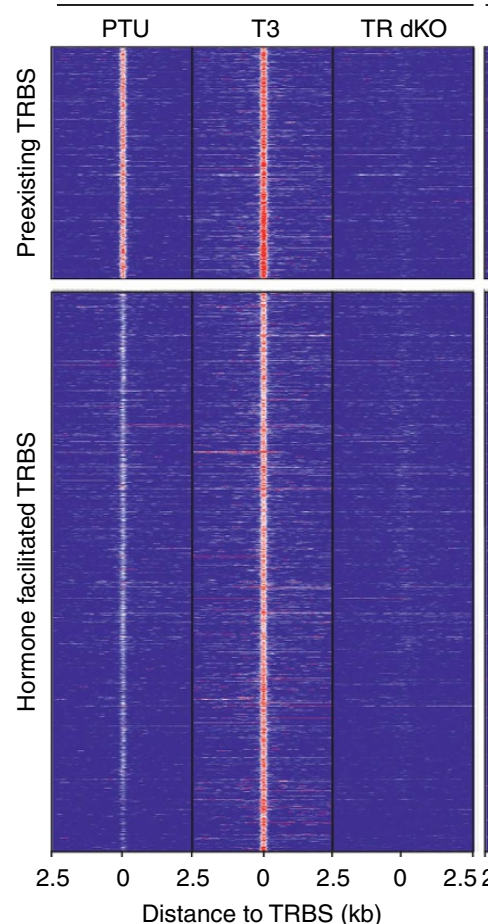

DNase-seq

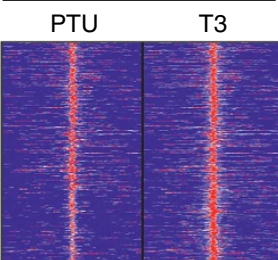

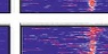

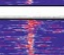

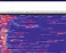
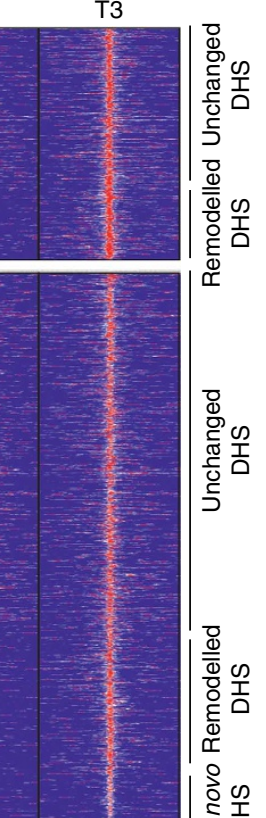

e

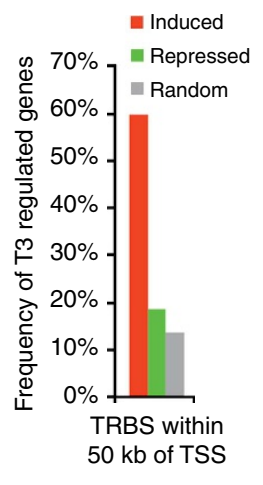

g

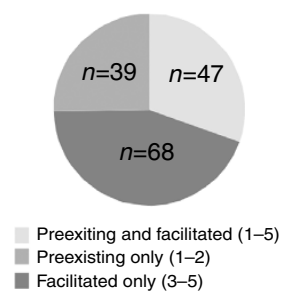

f

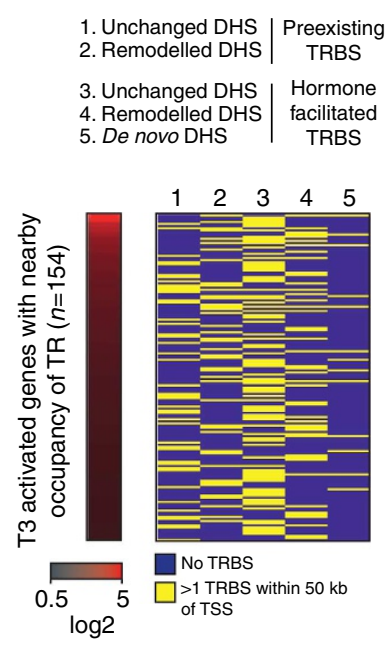

Figure 3 | Thyroid hormone-induced chromatin remodelling correlates with hormone-induced TR occupancy. ChIPs were performed on livers from mice treated with PTU, PTU + T3 and TR dKO. TR-binding sites (TRBS) were identified using HOMER with the TR ChIP-seq in TR dKO as a background control, a FDR threshold of 0.001 and a tag density threshold of 10 tags per peak. (a) The Venn diagram illustrates the overlap of TRBS in livers of PTU (yellow) and PTU plus T3-treated (blue) mice. Preexisting TR binding was defined by presence of TR peaks in absence and presence of T3, whereas hormone-facilitated TR binding was defined by a TR peak unique to T3 treatment. (b) Top panel, de novo motif analysis of TRBS in presence of T3. The most enriched motif is shown. Bottom panel, frequency of identified TRE in preexisting, hormone-facilitated and hormone-depleted TRBS. Broken line represents frequency of the TRE in all DNase-accessible regions of the genome in liver. (c) Distribution of TRBS within DHS regulated by T3 treatment. (d) Heat map illustrating TR ChIP-seq and DNase-seq tag distribution surrounding TRBS within the different categories of unchanged and T3-induced DHS. (e) Frequency of T3-regulated genes with at least one TRBS within $50 \mathrm{~kb}$ of TSS. Two hundred TSS from random genes were chosen as background control. (f) Heat map illustrating quantity of T3-activated genes with at least one TRBS (category 1-5) within $50 \mathrm{~kb}$ of TSS. Following TRBS categories are quantified:

(1) Preexisting TRBS within unchanged DHS. (2) Preexisting TRBS within remodelled TRBS. (3) Hormone-facilitated TRBS within unchanged DHS.

(4) Hormone-facilitated DHS within remodelled DHS. (5) Hormone-facilitated TRBS with de novo remodelled DHS. Genes are sorted from most induced and to most repressed by T3. (g) Number of T3-activated genes with a preexisting TRBS $(n=39,25 \%)$, hormone-facilitated TRBS ( $n=68,44 \%)$ or a combination of preexisting and hormone-facilitated TRBS $(n=47,31 \%)$ within $50 \mathrm{~kb}$ of TSS.

subpopulation of potential TRBS. However, the overall RXR ChIP-seq tag density of the RXR-binding sites not occupied by TR is significantly lower compared with TR-RXR co-occupied sites (Supplementary Fig. 3d), suggesting that the TR antibody fails to detect these potential binding sites as a result of low receptor occupancy and less likely due to possible epitope masking. Moreover, the TRE frequency is considerably lower at T3-remodelled DHS occupied by RXR without TR compared with RXR sites co-occupied by TR (Supplementary Fig. 3e), suggesting that many of the potential additional TRBS identified using RXR ChIP-seq may be weak indirect TRBS from tethering to other TFs. Thus chromatin in liver tissue may indeed harbour a relative low number of TRBS. In agreement, $<5,000$ TR molecules per cell has been estimated to be expressed in murine liver tissue ${ }^{27}$ in contrast to, for example, $>60,000 \mathrm{GR}$ molecules per cell in liver $^{28}$, suggesting that the relative low number of TRBS reflects low abundance of the receptor. In addition, recent studies probing dexamethasone-dependent GR occupancy of accessible chromatin in liver tissue using a combination of three different antibodies also demonstrates a 
relative low frequency of de novo-remodelled DHS occupied by GR $(13 \%)$ at a given point of time ${ }^{17}$. Importantly, similar trends are seen for ER and AR occupancy of remodelled chromatin in MCF7 and LNCaP cells, respectively ${ }^{25}$, inferring that low receptor occupancy of remodelled DHS is not necessarily a technical issue related to the antibody but may be associated to the dynamic behaviour of nuclear receptors (see below) or secondary effects of hormone treatment.

T3-dependent and independent TR occupancy of chromatin. The presented DNAse-seq and ChIP-seq against endogenous TR implies considerable ligand-dependent recruitment of TR to chromatin. Preexisting and hormone-facilitated TRBS are primarily located within unchanged and differentially remodelled DHS and almost no TR binding is found within DHSs reduced in accessibility as a consequence of T3 treatment (Fig. 3c). Interestingly, $10 \%$ of hormone-facilitated TR recruitment localize to de novo-remodelled chromatin, demonstrating that ligand-dependent TR recruitment to chromatin may function to regulate access to the chromatinized DNA template at a subset of TRBS (Fig. 3c). Since the vast majority of TRBS are found within unchanged and T3-induced accessible DHSs, we divided TRBS into hormone-preexisting and -facilitated binding sites and subdivided each group according to interaction with unchanged or induced DHSs (Fig. 3d). Supplementary Figure 4a shows overall DNase-seq tag density of the different types of TRBS. Very few de novo DHSs was identified within preexisting TRBS, illustrating that unliganded TR primarily occupies accessible chromatin. Interestingly, within all groups of TRBS, T3 increases overall TR recruitment to chromatin, even though it is most pronounced at the hormone-facilitated TRBS (Supplementary Fig. 4b). Also the TR ChIP-seq signal in hypothyroid condition is higher within preexisting TRBS compared with hormone-facilitated TRBS (Supplementary Fig. 4b). Similar observation is seen for RXR ChIP-seq signal at TRBS occupied by RXR (Supplementary Fig. 4c). This demonstrates that hormone generally stimulates TR occupancy of chromatin, which agrees with recent reports showing hormone-facilitated recruitment of ectopically expressed $\mathrm{TR}^{22}$. Interestingly, $\mathrm{T} 3$ does not lead to increased $\mathrm{TR}$ accumulation in the nucleus (Supplementary Fig. 2a), suggesting that increased chromatin occupancy is not simply explained by increased protein concentration.

To identify potential mechanisms regulating selective TR recruitment to preexisting TRBSs and insignificant binding to hormone-facilitated TRBSs in hypothyroid condition, we mined the TRBSs for enrichment and motif strength of the DR4 element identified. Interestingly, TRBSs within accessible chromatin bound by unliganded TR have a higher frequency of TRE and higher TRE motif score (Supplementary Fig. 5a,b, orange) compared with accessible TRBS not bound by unliganded TR (Supplementary Fig. 5a,b, grey). Also, TRBSs bound by unliganded TR have a higher degree of accessibility in hypothyroid condition compared with unbound accessible TRBSs (Supplementary Fig. 5c). Notably, de novo remodelled TRBSs have the highest frequency of TREs and the highest TRE motif score (Supplementary Fig. 5a,b, red). Thus, selective TR recruitment to chromatin is determined by the strength and frequency of the response element. Here unliganded TR preferentially occupies accessible chromatin with a strong consensus motif. Less accessible chromatin with weaker binding motifs display low occupancy by TR. Interestingly, a highly conserved motif is needed at sites where TR is able to penetrate inaccessible chromatin. Binding leads to remodelling through recruitment of co-activator complexes. Consequently, unliganded TR will not occupy these types of response elements.
T3-activated but not -repressed genes associated with TRBS. To gain insight to the mechanism regulating TR-dependent gene transcription, we mapped TRBS in vicinity to T3-regulated genes. Sixty per cent of T3-induced genes have at least one TRBS within $50 \mathrm{~kb}$ of the TSS (Fig. 3e). In contrast, T3-repressed genes show little enrichment of nearby TRBS, suggesting that liganded TR is preferentially associated with gene activation (Fig. 3e). We observe a similar trend for a range of regions between 5 and $200 \mathrm{~kb}$ of TSS from T3-regulated genes (Supplementary Fig. 5d). This agrees with the absence of TR-binding motifs in T3-regulated DHS near T3-repressed genes (Fig. 1e). Collectively, 154 T3-activated genes have nearby occupancy of TR (Supplementary Table 2). This list of genes likely constitutes direct TR targets in liver and gene ontology analysis indicates direct TR regulation of genes involved in fatty acid, steroid and cholesterol metabolism (Supplementary Table 3). Interestingly, TR-activated genes have nearby binding of multiple different TRBS described above (Fig. 3f). Importantly, two-thirds of the T3-activated genes are associated with hormone-facilitated TR recruitment to chromatin (Fig. 3g), suggesting that hormonefacilitated recruitment of $\mathrm{TR}$ to chromatin contributes to the regulation of a majority of direct TR target genes.

TR has been reported to bind DNA as a mono, homodimer or heterodimer with $\mathrm{RXR}^{1}$. $\mathrm{RXR} \alpha$ co-occupies $72 \%$ of TRBS in hypothyroid condition and $60 \%$ of TRBS in hyperthyroid condition (Supplementary Fig. 3f), suggesting that the majority of TR interacts with chromatin as a heterodimer with $\mathrm{RXR} \alpha$. This observation contradicts recent TR ChIP-seq data using overexpression of $\mathrm{TR}$, where $<40 \%$ of $\mathrm{TR}$ was reported to overlap with $\mathrm{RXR}^{22}$. However, the study showed that TR co-occupancy of RXR had a higher correlation with nearby T3-regulated gene transcription compared with TRBS without RXR. In agreement, we also observed a higher frequency of T3-induced genes with nearby occupancy of TR-RXR compared with TRBS without presence of RXR (Supplementary Fig. 5e). Neither TR-RXR co-occupancy nor TR occupancy without RXR correlates with nearby repression, implying that TR homodimers are not specifically involved in gene repression.

Activation of TR target genes through multiple mechanisms. Idh3, Gpd2 and $P d p 2$ represent direct TR target genes with nearby occupancy of TR in hypothyroid condition and thus potentially actively repressed by $\mathrm{TR}$ in that state (Fig. $4 \mathrm{a}-\mathrm{c}$ and Supplementary Table 2). In agreement, previous studies have shown that $\operatorname{Idh} 3 a$ is de-repressed in a TR $\beta$ KO model under hypothyroid condition ${ }^{29}$ and mutation of NCoR-disrupting interaction with TR de-represses $G p d 2$ and $I d h 3 a$ transcription in hypothyroid animals ${ }^{30}$. Activation of $G p d 2$ and $P d p 2$ transcription is not associated with considerable change of TR occupancy and chromatin is not significantly remodelled (Fig. 4a,b). At the Gpd2 and Pdp2 TRBSs, NCoR and HDAC3 occupancy is reduced in response to T3 (Fig. 4e,f); however, CBP is present at the TRBSs in the absence and the presence of ligand (Fig. 4g). This suggests that activation of Gpd2 and $P d p 2$ is coupled with co-repressor disassociation but in contrast to the bimodal switch model, co-activator pre-occupies TRBS in the absence of hormone and continues to occupy chromatin in the presence of hormone.

Idh3 represents a gene harbouring different types of TRBS in vicinity of the TSS (Fig. 4c). Close to the TSS, TR occupies a TRBS in the absence of hormone (Fig. $4 \mathrm{c}, I d h 3$, promoter). In addition, at a $8 \mathrm{~kb}$ upstream TRBS TR is hormone dependently recruited to chromatin associated with increased chromatin remodelling (Fig. 4c, Idh3, enhancer). NCoR and HDAC3 dissociate from the promoter in response to T3 treatment (Fig. 4e,f) and CBP is recruited to the enhancer (Fig. $4 \mathrm{~g}$ ), illustrating that a combination 
a

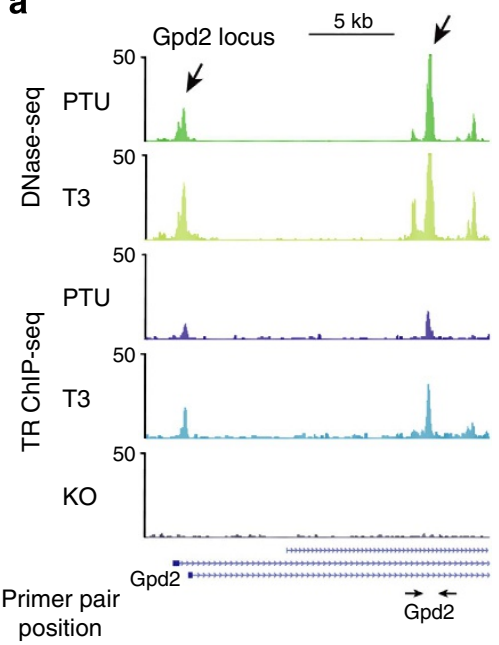

b
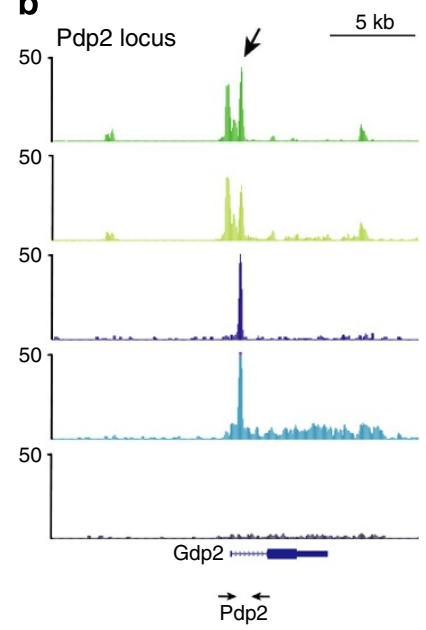

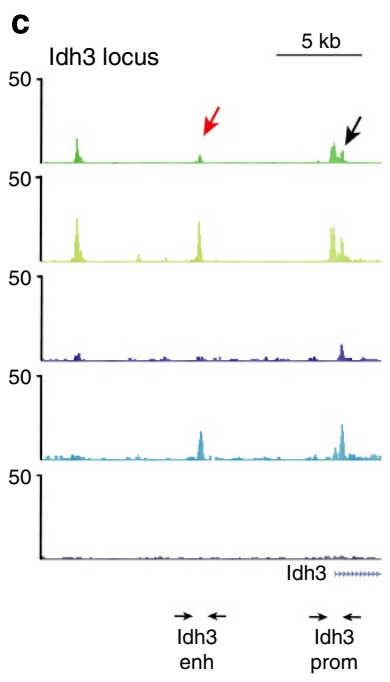

d

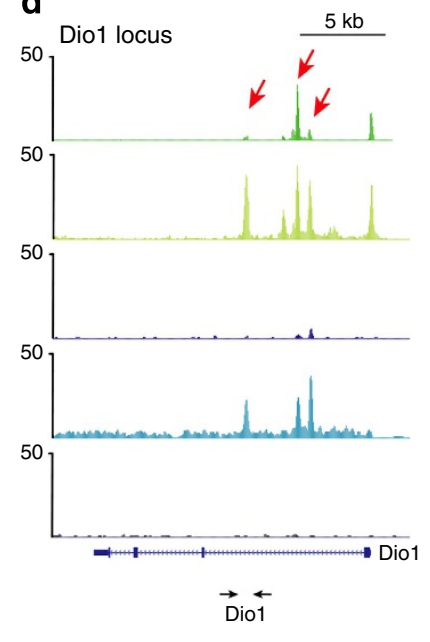

e

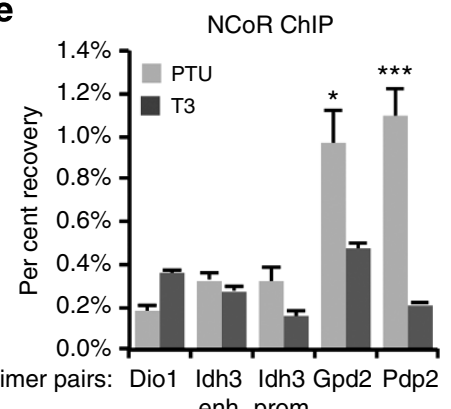

f

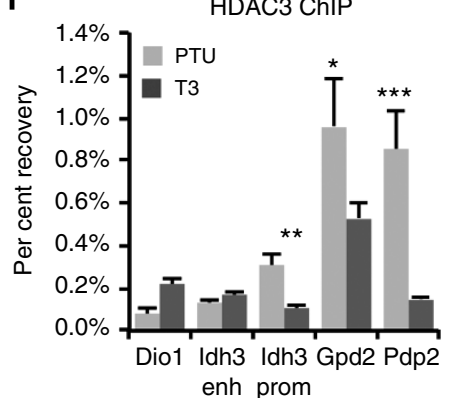

g

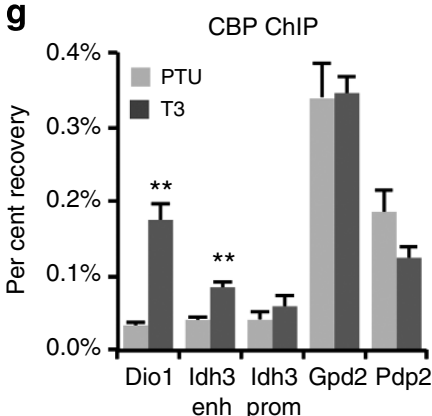

Figure 4 | Recruiment of co-repressors and co-activators to ligand-dependent and ligand-independent TRBS. (a-d) Examples of T3-activated genes (Idh3, Gpd2, Dio1 and Pdp2) harbouring nearby TRBS with preexisting TR occupancy (black arrows) and hormone-facilitated TR occupancy (red arrows). (e-g) ChIP against NCoR (e), HDAC3 (f) and CBP (g) at TRBS indicated in a-d. ChIPs were performed on livers from mice treated with PTU and PTU + T3. Error bars indicate s.e.m. with $n=4$ in each group. ${ }^{\star} P<0.05$. ${ }^{\star \star} P<0.01 .{ }^{\star \star \star} P<0.001$ ( $t$-test).

of co-repressor loss at one site and co-activator gain at another site may drive activation of transcription.

Interestingly, Dio1 represents a class of genes, where all nearby TR binding is ligand-dependent and TR recruitment leads to dramatic remodelling of the TRBSs (Fig. 4d). Presence of T3 leads to increased recruitment of co-activator CBP (Fig. 4g), whereas NCoR and HDAC3 occupancy remains unchanged at the TRBS (Fig. 4e,f). Thus, Dio1 is not a candidate for considerable direct repression by TR, concretely illustrating multiple mechanisms by which thyroid hormones regulate gene expression programmes. In agreement, disruption of NCoR-HDAC3 interaction have been reported to have little effect on Diol transcription in liver ${ }^{31}$.

Absence of TR FPs within chromatin. Early studies showed that at certain sites, liganded as well as unligand TR interaction with chromatin is associated with a DNase-accessible region and a FP corresponding to TR bound directly to $\mathrm{DNA}^{3}$. To gain genomewide insight to direct TR interaction with chromatin within identified TRBS, we carried out deep sequencing ( $>100$ million uniquely aligned tags) of DNase tag libraries from liver of hypo or hyperthyroid mice. Interestingly, we could not find evidence for significant genome-wide FPs protected from DNase cleavage at TR-bound TREs in comparison to unbound TREs (Fig. 5a, top). Similarly, comparing the DNase cut profiles between the PTU and T3 conditions did not identify nuclease protection at preexisting TR-bound TREs (Fig. 5b). This indicates that the TR interaction with chromatin generally does not create a detectable TR FP. Moreover, the DNase cleavage pattern at the TRE is the same irrespective of TR presence or absence on chromatin and is essentially identical to the cleavage pattern for naked DNA (Fig. 5a, bottom). This demonstrates that chromatinbound TR does not leave a unique DNase cleavage signature and agrees well with recent findings, demonstrating that other steroid hormone receptors such as $\mathrm{AR}^{32}, \mathrm{ER}$ and $\mathrm{GR}^{33} \mathrm{FP}$ poorly and do not create DNase cleavage signatures different from DNase cleavage signatures on naked DNA. In contrast, and as a control for the DNase-seq quality, we found considerable protection from DNase cleavage at previously described ChIP-seq identified bindings sites for CTCF, E2F4 and several liver-enriched TFs, including $\mathrm{C} / \mathrm{EBP}^{34}$ (Fig. 5c). CTCF and C/EBP have previously been shown to FP genome wide ${ }^{35,36}$. Collectively, our findings demonstrate that TR occupancy is generally not associated with a FP in chromatin and this may likely be a general phenomenon for steroid hormone receptors irrespective of their ability to repress transcription.

\section{Discussion}

Unlike steroid hormone receptors such as GR, AR and PR, unliganded TR is localized to the nucleus, where the receptor interacts with chromatin to repress transcription. Over the last two decades, transcriptional regulation by TR has been described by a model, wherein TR is bound to chromatin in the absence of ligand, recruits co-repressor complexes and represses transcription. On ligand binding, the receptor undergoes a hormonedependent conformational change, leading to disassociation of co-repressors and recruitment of co-activators. This model originates from early pioneering studies on steroid hormone receptors, where TR was demonstrated to interact with chromatin 
a
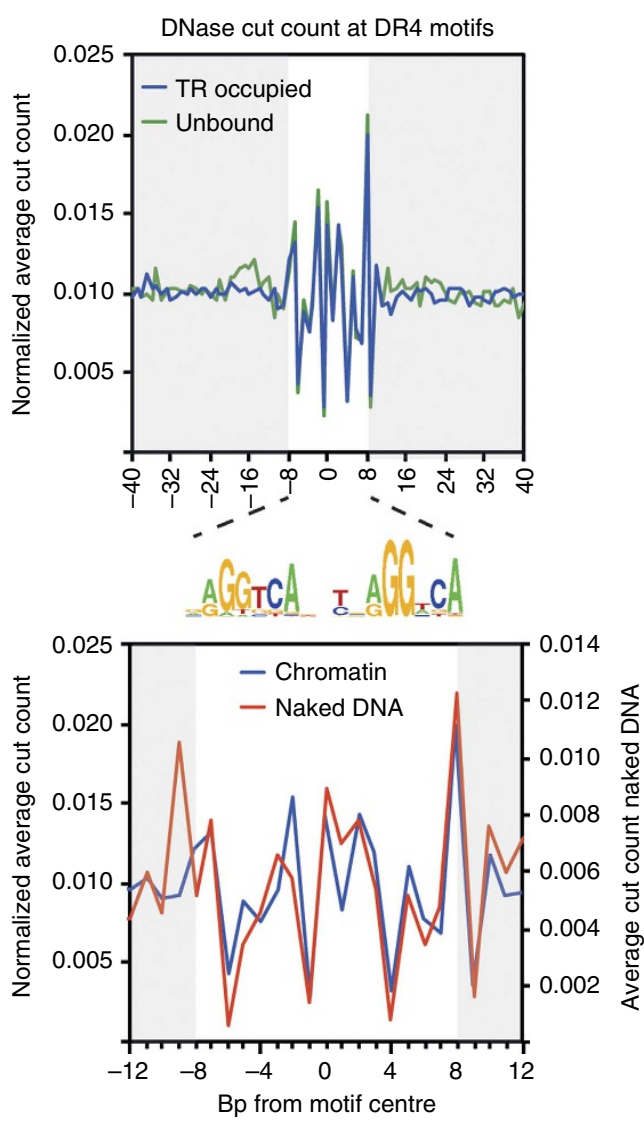

b

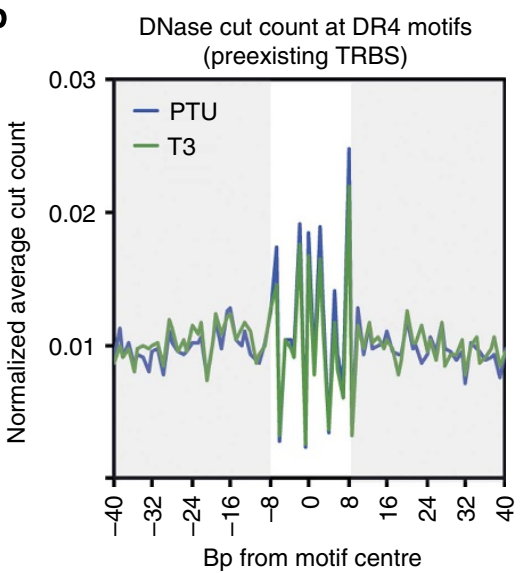

C
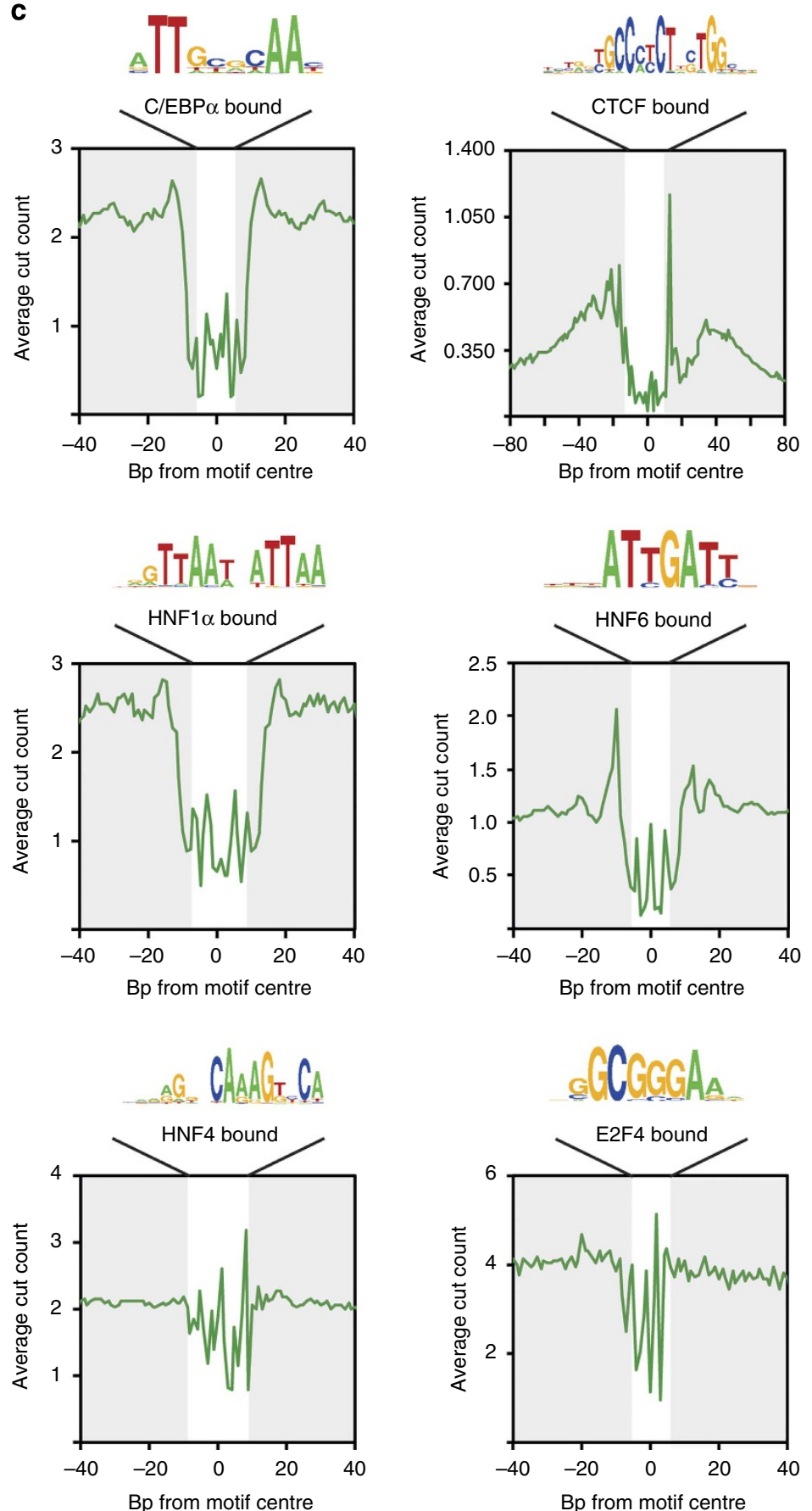

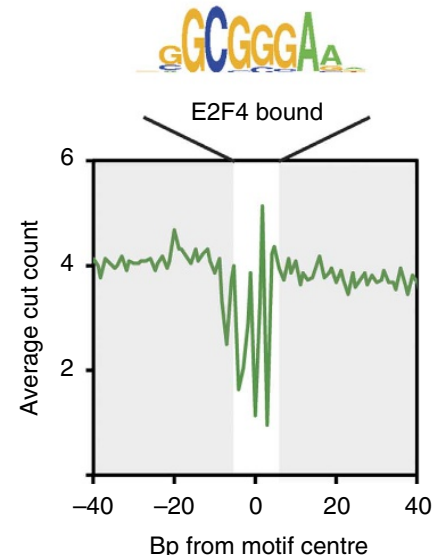

Figure 5 | Absence of DNase protection at DR4 motifs within TRBS. (a) Top panel, average DNase cut analysis of DR4 motifs enriched at TRBS within TR-bound (blue) and -unbound (green) regions of chromatin. DNase-seq library is from livers isolated from hyperthyroid mice. Average DNase cut count analysis of DR4 motifs at TR-bound (blue) and within genome-wide naked DNA from human IMR90 cells ${ }^{61}$ (bottom panel). (b) Average DNase cut count analysis of DR4 motifs within preexisting pre-accessible TRBS under hypo (PTU, blue) or hyperthyroid (T3, green) condition. (c) Average DNase cut count analysis of motifs within C/EBP $\alpha, C T C F, H N F 1 A, H N F 6, H N F 4$ and E2F4-binding sites identified previously in liver ${ }^{34}$. DNase-seq library is from livers isolated from hyperthyroid mice.

both in the presence and the absence of hormone ${ }^{37,38}$. Interaction was shown to be associated with increased DNase hypersensitivity and bound TR was demonstrated to $\mathrm{FP}^{3}$, leading to the general assumption that TR is constitutively bound to chromatin. Subsequent identification of transcriptional co-activators and co-repressors interacting with TR led to the widely accepted co-factor switch model described above (Supplementary Fig. 6a). This predicts that genome-wide footprinting analysis may be used to identify TREs. Unexpectedly, we find that thyroid hormone induces a considerable number of genome-wide de novo DNase hypersensitive sites harbouring putative TRBS (DR4), indicating that TR can be recruited to chromatin in a ligand-dependent manner, resulting in a DNase hypersensitive site. ChIP-seq against endogenous TR supports a model where TR can be recruited to chromatin in a ligand-dependent manner (Supplementary Fig. 6c,d). Importantly, we show that induced 
chromatin remodelling, including de novo remodelling, is associated with thyroid hormone-induced (and not repressed) transcription of nearby genes, demonstrating that liganddependent recruitment of TR and resulting chromatin remodelling are associated with thyroid hormone-activated gene transcription.

TR ChIP on a few promoters $^{39}$ and recent genome-wide ChIP-seq studies of exogenous expressed $\mathrm{TR}^{22}$, support our findings that $\mathrm{TR}$ can be recruited to chromatin in a ligand-dependent manner. Also, mutation of NCoR disrupting TR-NCoR interaction and mutation of NCoR abolishing HDAC3 interaction only leads to de-repression of a subset of TR-regulated genes $^{30,31}$, and repression of positively regulated TR target genes is only observed for a subset of thyroid hormone-activated genes $^{29}$. These findings collectively suggest an alternative mechanism for TR-regulated gene transcription, wherein ligand-dependent recruitment of TR to the genome can result in de novo remodelling of chromatin- and dynamic-assisted loading of transcriptional co-regulators ${ }^{20,40}$ (Supplementary Fig. 6d). Notably, the previously described dynamic mobility of TR in living cells ${ }^{41}$ is also supportive of the model described here. This mechanism is shared with a subset of GR, PR and AR chromatin interaction events ${ }^{17,18,25,42,43}$. Moreover, and in agreement with observations from other steroid hormone receptors ${ }^{17}$, repressed transcription as a consequence of hormone treatment is associated with nearby regions with reduced chromatin accessibility. These regions are not enriched for hormone receptor binding sites and are thus possibly regulated by indirect mechanisms such as transcriptional coregulator sequestering. In addition, TR ChIP-seq does not show enrichment of TRBS near repressed genes in agreement with tagged TR ChIP-seq studies from neuronal cells ${ }^{24}$. A recent study using TR overexpression suggested that hormone-facilitated gene repression is linked to reduced TR occupancy ${ }^{22}$. We do not find significant reduction of endogenous TR occupancy in response to hormone.

Although stable unliganded TR interaction with chromatin predominates in current models, several early studies indicated that TR does not necessarily FP on chromatin in the absence of ligand ${ }^{44,45}$. Using a genome-wide footprinting strategy, we show that, in contrast to several factors (for example, C/EBP and HNF6) TR generally does not footprint (Fig. 5a,b). Importantly, we did not find FPs at TRBS in the hypothyroid condition, suggesting that repressive TR interaction with chromatin is dynamic (Supplementary Fig. 6b). Lack of footprinting may be a general phenomenon for steroid hormone receptors ${ }^{32,33}$ and may be credited to the highly dynamic mechanisms of their interaction with chromatin $20,33,41,46$.

Collectively, we demonstrate that TR is recruited to chromatin by multiple mechanisms, including hormone-independent interactions with TREs for unliganded TR and ligand-induced TR recruitment to chromatin. This leaves a very important question of how TR discriminates between these two extremes, especially in hypothyroid conditions. Motif analysis suggests that the presence and strength of the TR-binding motif (DR4) within accessible chromatin is an important determinant regulating TR occupancy of chromatin in absence of T3, where unliganded TR preferentially occupy regions with strong TREs. In contrast, when T3 is available, TR is able to occupy accessible chromatin harbouring less stringent TR-binding motifs. Here ligandfacilitated recruitment to pre-accessible chromatin may be orchestrated by cooperation with pre-bound $\mathrm{TFs}^{17,47-49}$. Cooperation likely requires liganded TR and will only be favourable under such conditions. Alternatively binding to weak TREs may be regulated by nuclear distribution of TR. Previous studies have shown that hormone changes continuous shuttling of TR between cytoplasm and nucleus as well as redistribution of $\mathrm{TR}$ in the nucleus ${ }^{2,41}$. Reorganization of TR may increase subnuclear TR concentration and drive hormone-dependent recruitment to specific sites of the genome. Changing local TF concentration relative to the amount of binding sites have been shown to rewire transcriptional networks, likely determined by differential affinity of TF for its degenerated binding sequence ${ }^{50}$. Thus, binding of TR to particular TRBS may be driven by specific receptor-DNA affinity, and increasing TR concentration may therefore increase occupancy of particular hormone-sensitive TRBS. Such hormone-sensitive TRBS may work similar to the reported glucocorticoid hypersensitive sites ${ }^{51}$. Importantly, however, we do not observe considerable increase in nuclear accumulation of TR $\beta$ probed by IP (Supplementary Fig. 2a).

Interestingly, at a subset of TRBS and in the presence of T3, TR is able to penetrate an inaccessible chromatin configuration and induce de novo chromatin remodelling. Here a strong TRE is required (Supplementary Fig. 5b) together with the ability to interact with transcriptional co-activators (Fig. 4g). Consequently, these TRBS cannot be accessed by unliganded TR. Selectivity to these sites may also be driven by cooperation with other TFs, and remodelling proteins to remodel chromatin and promote TR occupancy as seen for other steroid hormone receptors such as $\mathrm{GR}^{17,25,49,52}$. Collectively, this opens a series of new questions regarding specificity and importance of ligand-dependent TR binding to chromatin and may facilitate additional molecular insights into the basis of TR mutations in disease.

\section{Methods}

Animal work. Wild-type mice were treated with PTU (0.15\%; Harlan Teklad, Cat\#TD.95125) for 3 weeks and then divided into two groups. One group was treated with PTU to maintain hypothyroid status and the other group was treated with PTU together with i.p. injection of T3 injection at a dose of $10 \mu \mathrm{g}$ per $100 \mathrm{~g}$ body weight for 5 days to make mice hyperthyroid. The animal study was carried out according to the protocol approved by the National Cancer Institute Animal Care and Use Committee.

RNA expression analysis. Total RNA ( $250 \mathrm{ng}$ ) was reverse transcribed priming with a T7 oligo(dT) primer, followed by second strand complementary DNA synthesis. Biotin-labelled complementary RNA was produced by in vitro transcription, and hybridized to MouseRef- 8 v2.0 Expression BeadChip arrays according to the manufacturer's instructions (Illumina). Three biological replicates (independent mice) were used for each condition (PTU and T3). RNA was hybridized to the Illumina MouseRef- 8 v2.0 Expression BeadChip and raw data were extracted with Illumina BeadStudio software. Raw probe intensities were converted to expression values using the lumi package ${ }^{53}$ in Bioconductor with background correction, variance stabilization by vst and quantile normalization. Differential expression between PTU and T3 conditions was assessed by an empirical Bayes analysis of a linear model using the limma package ${ }^{54}$ in Bioconductor. False discovery rates were calculated with the Benjamini and Hochberg method, and differentially expressed genes were defined with a false discovery rate (FDR) cutoff of 0.3 and a fold change of 1.5 .

DNase digestion of chromatin. Livers were isolated from mice and nuclei were immediately purified $^{21}$ and resuspended in buffer A ( $15 \mathrm{~mm}$ Tris- $\mathrm{HCl} \mathrm{pH} \mathrm{8.0,}$ $15 \mathrm{~mm} \mathrm{NaCl}, 60 \mathrm{~mm} \mathrm{KCl,} 1 \mathrm{~mm}$ EDTA, $0.5 \mathrm{~mm}$ EGTA, $0.5 \mathrm{~mm}$ Spermidine and protease inhibitors) in a final concentration of 10 million nuclei per $\mathrm{ml}$. DNase digestions were performed by adding $100 \mu \mathrm{l} \times 10$ digestion buffer $\left(60 \mathrm{mM} \mathrm{CaCl}_{2}\right.$ and $750 \mathrm{~mm} \mathrm{NaCl}$ ) containing various concentrations (0 U-100 U) of DNase I (Sigma). Digestions were incubated for $3 \mathrm{~min}$ at $37^{\circ} \mathrm{C}$ and reactions were terminated by addition of one volume of stop buffer $(50 \mathrm{~mm}$ Tris- $\mathrm{HCl}, 100 \mathrm{~mm}$ $\mathrm{NaCl}, 0.1 \%$ SDS, $100 \mathrm{~mm}$ EDTA and $50 \mu \mathrm{g} \mathrm{ml}^{-1}$ Proteinase $\mathrm{K}$ (Ambion)). Digested chromatin was incubated at $55^{\circ} \mathrm{C}$ for $2 \mathrm{~h}$ and stored at $4{ }^{\circ} \mathrm{C}$ until further use. DNase I digestion efficiency was evaluated by quantitative PCR and samples with optimal digestion efficiency were incubated with $90 \mu \mathrm{g} \mathrm{ml}^{-1}$ RNase A (Sigma) for $30 \mathrm{~min}$ at $37^{\circ} \mathrm{C}$ before $50-500 \mathrm{bp}$ DNA fragments were purified using ultracentrifugation.

Chromatin IP. Frozen livers were homogenized in PBS containing 1\% formaldehyde, incubated $10 \mathrm{~min}$ at room temperature and quenched with $0.125 \mathrm{M}$ glycine. For HDAC3, CBP and NCoR IP's an additional crosslinker, disuccinimidyl glutarate, was used prior to formaldehyde fixation. Crosslinked cells were washed 
in PBS, resuspended in lysis buffer $(0.1 \%$ SDS, $1 \%$ Triton X-100, $150 \mathrm{~mm} \mathrm{NaCl}$ and $20 \mathrm{~mm}$ HEPES pH 7.6) and sonicated using a Bioruptor (Diagenode). Chromatin was immunoprecipitated using monoclonal antibody $\mathrm{C} 1$ which preferentially recognize TR $\beta 1$, NCoR (Abcam, ab24552), HDAC3 (Santa Cruz, sc-11417), RXR (Santa Cruz, sc-553) and CBP (Santa Cruz, sc-369) and Protein A/G agarose beads (Santa Cruz, sc-2003) over night at $4{ }^{\circ} \mathrm{C}$. IPs were performed in $1 \mathrm{ml}$ aliquots using $3 \mu \mathrm{g}$ of indicated antibodies. Immunocomplexes were washed extensively and chromatin was eluted and decrosslinked over night at $65^{\circ} \mathrm{C}$. DNA was subsequently phenol/chloroform purified and ethanol precipitated.

Illumina sequencing. ChIP- or DHS-isolated DNA (10 ng) was used in preparation of standard Illumina sequencing libraries. In brief, DNA fragments were endrepaired, adenylated and ligated to adapter sequences. Following gel purification, index sequences were introduced by PCR. Library cluster generation and singleend sequencing was performed on the Ilumina platform. Reads were aligned with bwa 0.5 .9 -r16 (ref. 55) to the $\mathrm{mm} 9$ reference genome and duplicates were marked with picard version 1.92 (ref. 56).

DNase-seq and ChIP-seq analysis. Replicate concordant DHS were identified using the Hotspot algorithm ${ }^{57}$. Differentially accessible DHS were scored using DESeq $^{58}$ from two biological replicates at adjusted $P$ value $<0.05$. TR ChIP-seq enriched regions were identified by HOMER ${ }^{59}$ using a TR ChIP-seq from TR KO as a background control, a FDR threshold of $0.1 \%$ and a tag density threshold of 10 . RXR-ChIP-enriched regions were identified using input chromatin as control, a FDR threshold of $0.1 \%$ and a tag density threshold of 20. Enriched DNA motifs at DHS- and TR-enriched regions were identified using HOMER and heatmaps of ChIP-seq and DNase-seq data were generated using $\mathrm{MeV}^{60}$.

Analysis of DNase protection at TF-binding sites. From ultra deep sequencing of DNase-seq libraries, we generated DNase cut count profiles as described previously ${ }^{35}$. In short, because a single cut in a DNA segment by DNase I generates two end nucleotides, $5^{\prime}$ and $3^{\prime}$, we set the location to be the $5^{\prime}$ nucleotide to define cut count profile independently of the read strand from sequencing. If a read is mapped onto the forward strand, the cut site is defined as the -1 nucleotide (immediately $5^{\prime}$ ) from where the read starts. If a read is in the reverse strand, we define the cut site to be the first base of the read.

Motifs within TF ChIP-seq peaks were extracted using HOMER and the average DNase cut count was evaluated at base pair resolution centred on TF motifs. Coordinates from liver-enriched TFs were based on previously published ChIP-seq data $^{34}$.

\section{References}

1. Cheng, S. Y., Leonard, J. L. \& Davis, P. J. Molecular aspects of thyroid hormone actions. Endocr. Rev. 31, 139-170 (2010).

2. Baumann, C. T., Maruvada, P., Hager, G. L. \& Yen, P. M. Nuclear cytoplasmic shuttling by thyroid hormone receptors. multiple protein interactions are required for nuclear retention. J. Biol. Chem. 276, 11237-11245 (2001).

3. Wong, J., Shi, Y. B. \& Wolffe, A. P. A role for nucleosome assembly in both silencing and activation of the Xenopus TR beta A gene by the thyroid hormone receptor. Genes Dev. 9, 2696-2711 (1995).

4. Tong, G. X., Jeyakumar, M., Tanen, M. R. \& Bagchi, M. K. Transcriptional silencing by unliganded thyroid hormone receptor beta requires a soluble corepressor that interacts with the ligand-binding domain of the receptor. Mol. Cell Biol. 16, 1909-1920 (1996).

5. Chen, J. D., Umesono, K. \& Evans, R. M. SMRT isoforms mediate repression and anti-repression of nuclear receptor heterodimers. Proc. Natl Acad. Sci. USA 93, 7567-7571 (1996)

6. Horlein, A. J. et al. Ligand-independent repression by the thyroid hormone receptor mediated by a nuclear receptor co-repressor. Nature 377, 397-404 (1995)

7. Lin, B. C., Hong, S. H., Krig, S., Yoh, S. M. \& Privalsky, M. L. A conformational switch in nuclear hormone receptors is involved in coupling hormone binding to corepressor release. Mol. Cell Biol. 17, 6131-6138 (1997).

8. Perissi, V. et al. Molecular determinants of nuclear receptor-corepressor interaction. Genes Dev. 13, 3198-3208 (1999)

9. Chen, H. et al. Nuclear receptor coactivator ACTR is a novel histone acetyltransferase and forms a multimeric activation complex with P/CAF and CBP/p300. Cell 90, 569-580 (1997).

10. Fondell, J. D., Ge, H. \& Roeder, R. G. Ligand induction of a transcriptionally active thyroid hormone receptor coactivator complex. Proc. Natl Acad. Sci. USA 93, 8329-8333 (1996).

11. Xu, L., Glass, C. K. \& Rosenfeld, M. G. Coactivator and corepressor complexes in nuclear receptor function. Curr. Opin. Genet. Dev. 9, 140-147 (1999).

12. Mullur, R., Liu, Y. Y. \& Brent, G. A. Thyroid hormone regulation of metabolism. Physiol. Rev. 94, 355-382 (2014).

13. Brent, G. A. Mechanisms of thyroid hormone action. J. Clin. Invest. 122, 3035-3043 (2012)
14. Astapova, I. \& Hollenberg, A. N. The in vivo role of nuclear receptor corepressors in thyroid hormone action. Biochim. Biophys. Acta 1830, 3876-3881 (2013).

15. Perissi, V., Jepsen, K., Glass, C. K. \& Rosenfeld, M. G. Deconstructing repression: evolving models of co-repressor action. Nat. Rev. Genet. 11, 109-123 (2010).

16. Mottis, A., Mouchiroud, L. \& Auwerx, J. Emerging roles of the corepressors NCoR1 and SMRT in homeostasis. Genes Dev. 27, 819-835 (2013).

17. Grontved, L. et al. C/EBP maintains chromatin accessibility in liver and facilitates glucocorticoid receptor recruitment to steroid response elements. EMBO J. 32, 1568-1583 (2013).

18. John, S. et al. Chromatin accessibility pre-determines glucocorticoid receptor binding patterns. Nat. Genet. 43, 264-268 (2011).

19. Miranda, T. B. et al. Reprogramming the chromatin landscape: interplay of the estrogen and glucocorticoid receptors at the genomic level. Cancer Res. 73, 5130-5139 (2013).

20. Voss, T. C. \& Hager, G. L. Dynamic regulation of transcriptional states by chromatin and transcription factors. Nat. Rev. Genet. 15, 69-81 (2014).

21. Grontved, L. et al. Rapid genome-scale mapping of chromatin accessibility in tissue. Epigenetics Chromatin 5, 10 (2012).

22. Ramadoss, P. et al. Novel mechanism of positive versus negative regulation by thyroid hormone receptor betal (TRbetal) identified by genome-wide profiling of binding sites in mouse liver. J. Biol. Chem. 289, 1313-1328 (2014).

23. Ayers, S. et al. Genome-wide binding patterns of thyroid hormone receptor Beta. PLoS ONE 9, e81186 (2014).

24. Chatonnet, F., Guyot, R., Benoit, G. \& Flamant, F. Genome-wide analysis of thyroid hormone receptors shared and specific functions in neural cells. Proc. Natl Acad. Sci. USA 110, E766-E775 (2013).

25. He, H. H. et al. Differential DNase I hypersensitivity reveals factor-dependent chromatin dynamics. Genome Res. 22, 1015-1025 (2012).

26. Berrodin, T. J., Marks, M. S., Ozato, K., Linney, E. \& Lazar, M. A. Heterodimerization among thyroid hormone receptor, retinoic acid receptor, retinoid $\mathrm{X}$ receptor, chicken ovalbumin upstream promoter transcription factor, and an endogenous liver protein. Mol. Endocrinol. 6, 1468-1478 (1992).

27. Oppenheimer, J. H. \& Schwartz, H. L. Molecular basis of thyroid hormonedependent brain development. Endocr. Rev. 18, 462-475 (1997).

28. Wrange, O., Carlstedt-Duke, J. \& Gustafsson, J. A. Purification of the glucocorticoid receptor from rat liver cytosol. J. Biol. Chem. 254, 9284-9290 (1979).

29. Yen, P. M. et al. Effects of ligand and thyroid hormone receptor isoforms on hepatic gene expression profiles of thyroid hormone receptor knockout mice. EMBO Rep. 4, 581-587 (2003).

30. Astapova, I. et al. The nuclear corepressor, NCoR, regulates thyroid hormone action in vivo. Proc. Natl Acad. Sci. USA 105, 19544-19549 (2008).

31. You, S. H., Liao, X., Weiss, R. E. \& Lazar, M. A. The interaction between nuclear receptor corepressor and histone deacetylase 3 regulates both positive and negative thyroid hormone action in vivo. Mol. Endocrinol. 24, 1359-1367 (2010).

32. He, H. H. et al. Refined DNase-seq protocol and data analysis reveals intrinsic bias in transcription factor footprint identification. Nat. Methods 11, 73-78 (2014).

33. Sung, M. H., Guertin, M. J., Baek, S. \& Hager, G. L. DNase footprint signatures are dictated by factor dynamics and DNA sequence. Mol. Cell 56, 275-285 (2014).

34. Faure, A. J. et al. Cohesin regulates tissue-specific expression by stabilizing highly occupied cis-regulatory modules. Genome Res. 22, 2163-2175 (2012).

35. Siersbaek, R. et al. Molecular architecture of transcription factor hotspots in early adipogenesis. Cell Rep. 7, 1434-1442 (2014).

36. Boyle, A. P. et al. High-resolution genome-wide in vivo footprinting of diverse transcription factors in human cells. Genome Res. 21, 456-464 (2011).

37. Perlman, A. J., Stanley, F. \& Samuels, H. H. Thyroid hormone nuclear receptor. Evidence for multimeric organization in chromatin. J. Biol. Chem. 257, 930-938 (1982).

38. Jump, D. B. \& Oppenheimer, J. H. Thyroid hormone receptor-containing fragment released from chromatin by deoxyribonuclease I and micrococcal nuclease. Science 209, 811-813 (1980).

39. Liu, Y., Xia, X., Fondell, J. D. \& Yen, P. M. Thyroid hormone-regulated target genes have distinct patterns of coactivator recruitment and histone acetylation. Mol. Endocrinol. 20, 483-490 (2006).

40. Voss, T. C. et al. Dynamic exchange at regulatory elements during chromatin remodelling underlies assisted loading mechanism. Cell 146, 544-554 (2011).

41. Maruvada, P., Baumann, C. T., Hager, G. L. \& Yen, P. M. Dynamic shuttling and intranuclear mobility of nuclear hormone receptors. J. Biol. Chem. 278, 12425-12432 (2003).

42. Tewari, A. K. et al. Chromatin accessibility reveals insights into androgen receptor activation and transcriptional specificity. Genome Biol. 13, R88 (2012) 
43. Ballare, C. et al. Nucleosome-driven transcription factor binding and gene regulation. Mol. Cell 49, 67-79 (2013).

44. Force, W. R. \& Spindler, S. R. 3,5,3'-L-triiodothyronine (thyroid hormone)induced protein-DNA interactions in the thyroid hormone response elements and cell type-specific elements of the rat growth hormone gene revealed by in vivo dimethyl sulfate footprinting. J. Biol. Chem. 269, 9682-9686 (1994).

45. Kim, S. W., Ahn, I. M. \& Larsen, P. R. In vivo genomic footprinting of thyroid hormone-responsive genes in pituitary tumor cell lines. Mol. Cell Biol. 16, 4465-4477 (1996)

46. Kumar, S., Saradhi, M., Chaturvedi, N. K. \& Tyagi, R. K. Intracellular localization and nucleocytoplasmic trafficking of steroid receptors: an overview. Mol. Cell Endocrinol. 246, 147-156 (2006).

47. Gertz, J. et al. Distinct properties of cell-type-specific and shared transcription factor binding sites. Mol. Cell 52, 25-36 (2013).

48. Pihlajamaa, P. et al. Tissue-specific pioneer factors associate with androgen receptor cistromes and transcription programs. EMBO J. 33, 312-326 (2014).

49. Biddie, S. C. et al. Transcription factor AP1 potentiates chromatin accessibility and glucocorticoid receptor binding. Mol. Cell 43, 145-155 (2011).

50. Brewster, R. C. et al. The transcription factor titration effect dictates level of gene expression. Cell 156, 1312-1323 (2014).

51. Reddy, T. E., Gertz, J., Crawford, G. E., Garabedian, M. J. \& Myers, R. M. The hypersensitive glucocorticoid response specifically regulates period 1 and expression of circadian genes. Mol. Cell Biol. 32, 3756-3767 (2012).

52. Hurtado, A., Holmes, K. A., Ross-Innes, C. S., Schmidt, D. \& Carroll, J. S. FOXA1 is a key determinant of estrogen receptor function and endocrine response. Nat. Genet. 43, 27-33 (2011).

53. Du, P., Kibbe, W. A. \& Lin, S. M. lumi: a pipeline for processing Illumina microarray. Bioinformatics 24, 1547-1548 (2008).

54. Smyth, G. K., Michaud, J. \& Scott, H. S. Use of within-array replicate spots for assessing differential expression in microarray experiments. Bioinformatics 21, 2067-2075 (2005)

55. Li, H. \& Durbin, R. Fast and accurate short read alignment with BurrowsWheeler transform. Bioinformatics 25, 1754-1760 (2009).

56. Li, H. et al. The sequence alignment/map format and SAMtools. Bioinformatics 25, 2078-2079 (2009).

57. Baek, S., Sung, M. H. \& Hager, G. L. Quantitative analysis of genome-wide chromatin remodelling. Methods Mol. Biol. 833, 433-441 (2012).

58. Anders, S. \& Huber, W. Differential expression analysis for sequence count data. Genome Biol. 11, R106 (2010).
59. Heinz, S. et al. Simple combinations of lineage-determining transcription factors prime cis-regulatory elements required for macrophage and B cell identities. Mol. Cell 38, 576-589 (2010).

60. Saeed, A. I. et al. TM4: a free, open-source system for microarray data management and analysis. Biotechniques 34, 374-378 (2003).

61. Lazarovici, A. et al. Probing DNA shape and methylation state on a genomic scale with DNase I. Proc. Natl Acad. Sci. USA 110, 6376-6381 (2013).

\section{Acknowledgements}

This work was supported by the Intramural Research Program of the National Institutes of Health (NIH), the National Cancer Institute (NCI) and the Center for Cancer Research (CCR). L.G. was supported by a research grant from the Lundbeck Foundation, the Danish Research Council and SDU2020. This study utilized the high-performance computational capabilities of the Biowulf Linux cluster at the National Institutes of Health, Bethesda, MD.

\section{Author contributions}

L.G. performed the ChIP-seq and DNase-seq experiments. D.W.K., L.Z. and J.W.P. performed the animal work and antibody validation. R.L.W., R.N. and Y.J.Z. performed Illumina sequencing and expression array analysis. L.G. and J.J.W. performed the data analysis with help from S.B., and M.S. L.G., J.J.W., P.S.M., G.L.H. and S.C. designed the experiments and wrote the manuscript.

\section{Additional information}

Accession codes: Gene expression microarray data have been deposited in the GEO database with accession number GSE65947. ChIP- and DNase-sequencing data have been deposited in the SRA database with accession number SRP055020.

Supplementary Information accompanies this paper at http://www.nature.com/ naturecommunications

Competing financial interests: The authors declare no competing financial interests.

Reprints and permission information is available online at http://npg.nature.com/ reprintsandpermissions/

How to cite this article: Grøntved, L. et al. Transcriptional activation by the thyroid hormone receptor through ligand-dependent receptor recruitment and chromatin remodelling. Nat. Commun. 6:7048 doi: 10.1038/ncomms8048 (2015). 